\title{
Comparison of Blood Oxygenation Level-Dependent fMRI and Provocative DSC Perfusion MR Imaging for Monitoring Cerebrovascular Reserve in Intracranial Chronic Cerebrovascular Disease
}

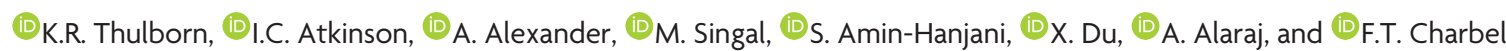
o를

\begin{abstract}
BACKGROUND AND PURPOSE: Loss of hemodynamic reserve in intracranial cerebrovascular disease reduces blood oxygenation leveldependent activation by fMRI and increases asymmetry in MTT measured by provocative DSC perfusion MR imaging before and after vasodilation with intravenous acetazolamide. The concordance for detecting hemodynamic reserve integrity has been compared.
\end{abstract}

MATERIALS AND METHODS: Patients $(n=40)$ with intracranial cerebrovascular disease and technically adequate DSA, fMRI and provocative DSC perfusion studies were retrospectively grouped into single vessels proximal to and distal from the circle of Willis, multiple vessels, and Moyamoya disease. The vascular territories were classified as having compromised hemodynamic reserve if the expected fMRI blood oxygenation level-dependent activation was absent or if MTT showed increased asymmetry following vasodilation. Concordance was examined in compromised and uncompromised vascular territories of each group with the Fischer exact test and proportions of agreement.

RESULTS: Extensive leptomeningeal collateral circulation was present in all cases. Decreased concordance between the methods was found in vascular territories with stenosis distal to but not proximal to the circle of Willis. Multivessel and Moyamoya diseases also showed low concordance. A model of multiple temporally displaced arterial inputs from leptomeningeal collateral flow demonstrated that the resultant lengthening MTT mimicked compromised hemodynamic reserve despite being sufficient to support blood oxygenation leveldependent contrast.

CONCLUSIONS: Decreased concordance between the 2 methods for assessment of hemodynamic reserve for vascular disease distal to the circle of Willis is posited to be due to well-developed leptomeningeal collateral circulation providing multiple temporally displaced arterial input functions that bias the perfusion analysis toward hemodynamic reserve compromise while blood oxygenation leveldependent activation remains detectable.

ABBREVIATIONS: ACA = anterior cerebral artery; AIF = arterial input function; BOLD = blood oxygenation level-dependent; CVD = cerebrovascular disease; $\mathrm{HR}=$ hemodynamic reserve; $\mathrm{LCC}=$ leptomeningeal collateral circulation; $\mathrm{MMD}=$ Moyamoya disease; $\mathrm{MVD}=$ multivessel disease; $\mathrm{PCA}=$ posterior cerebral artery; poa $=$ proportions of agreement; sMCA = superior division of middle cerebral artery; SVDd = single-vessel disease distal to the circle of Willis; SVDp = single-vessel disease proximal to the circle of Willis

C linical management of patients with chronic cerebrovascular disease (CVD) includes risk assessment for future stroke. Loss of hemodynamic reserve (HR) is one indicator of increased

Received May 29, 2017; accepted after revision November 7

From the Center for Magnetic Resonance Research (K.R.T., I.C.A., A.Alexander, M.S.) and Department of Neurological Surgery (S.A.-H., X.D., A.Alaraj, F.T.C.), University of Illinois Medical Center, Chicago, Illinois.

The authors acknowledge National Institutes of Health grants RO1 NS059745-01A1, RO1 NS3867601A1, and RO1 CA1295531A1, which provided partial salary support for K.R.T.

Please address correspondence to Keith R. Thulborn, MD, PhD, Center for Magnetic Resonance Research, Room 1307, OCC, M/C 707, University of Illinois at Chicago, 1801 West Taylor St, Chicago, IL 60612; e-mail: kthulbor@uic.edu

- Indicates open access to non-subscribers at www.ajnr.org

三 Indicates article with supplemental on-line tables.

http://dx.doi.org/10.3174/ajnr.A5515 risk, supporting more aggressive medical treatment and perhaps neurosurgical or neurovascular interventions. ${ }^{1-4}$ HR is assessed with many imaging modalities by the patterns of perfusion redistribution after vasodilation. While a perfusion study may or may not show asymmetry in baseline perfusion, vasodilation induced with acetazolamide may unmask perfusion asymmetries indicative of loss of HR. ${ }^{2}$

Blood oxygenation level-dependent (BOLD) contrast in fMRI arises from the increased local signal intensity from increased regional blood flow that occurs when neurons perform work. ${ }^{5}$ The role of fMRI for the evaluation of CVD has been reviewed. ${ }^{6} \mathrm{Be}-$ cause BOLD contrast requires an intact $\mathrm{HR}$, a paradigm that interrogates cortical areas in all vascular territories simultaneously can be used to detect regional $\mathrm{HR}$ in each vascular territory.

We have assessed the concordance between BOLD fMRI and 
provocative DSC perfusion MR imaging for assessing HR in a cohort of patients with CVD. A model of the multiple temporally displaced arterial input functions (AIFs) from well-developed leptomeningeal collateral circulation in chronic CVD is posited to explain the observed low concordance between these methods for detecting compromised HR.

\section{MATERIALS AND METHODS \\ Population}

This institutional review board-approved retrospective study comprised patients ( $n=40$; mean age, $48 \pm 17$ years; 24 women and 16 men) who underwent extensive evaluation for atherosclerotic CVD or Moyamoya disease, including DSA, MR imaging using DSC perfusion before and after vasodilation with acetazolamide, and fMRI with a customized HR assessment paradigm. Symptoms included $\geq 1$ instance of weakness $(n=23)$, parethesia $(n=11)$, difficulty speaking $(n=10)$, atypical headache $(n=7)$, dizziness $(n=4)$, visual disturbance $(n=3)$, and cognitive compromise $(n=2)$. Multiple risk factors for vascular disease were present in most subjects, including hypertension $(n=26)$, hyperlipidemia $(n=19)$, diabetes mellitus $(n=10)$, and smoking $(n=$ 23 ). Patients were selected from $>300$ cases reviewed in weekly clinical case review conferences attended by treating physicians across a 6-year period (June 2006 to June 2012). Although all patients were diagnosed as having CVD of varying severity, the cohort was selected with technically sound DSA, fMRI, and perfusion studies. Specifically, absence of head motion $(<1-\mathrm{mm}$ translational head motion in 3 orthogonal directions) was stipulated for all functional and perfusion studies. Only the first technically adequate examination was used for patients with multiple studies.

Four groups were formed on the basis of CVD distribution: single-vessel disease distal to the circle of Willis (SVDd, $n=7$; $56 \pm 12$ years of age; 2 women and 5 men, including 2 bypasses); single-vessel disease proximal to the circle of Willis with an intact posterior communicating artery ipsilateral to the stenotic internal carotid artery (SVDp, $n=9 ; 53 \pm 16$ years of age; 4 women and 5 men, including 1 bypass); multivessel intracranial disease not including Moyamoya disease (MVD, $n=10 ; 53 \pm 15$ years of age; 8 women and 2 men, including 3 bypasses); and Moyamoya disease (MMD) $(n=14 ; 38 \pm 16$ years of age; 10 women and 4 men, including 3 bypasses). Bypass grafts used anastomoses of the distal superficial temporal artery to the distal middle cerebral artery.

\section{Anatomic MR Imaging}

All MR imaging was performed at 3T (Signa; GE Healthcare, Milwaukee, Wisconsin) using routine axial high-resolution T2weighted FLAIR and gradient-echo imaging and 3D T1-weighted imaging before and after intravenous gadolinium contrast enhancement to exclude vascular territories with hemorrhage or encephalomalacic changes from previous infarctions. Acute stroke was excluded by diffusion-weighted imaging.

\section{Functional MR Imaging}

Whole-brain fMRI studies were performed using a published imaging protocol and customized paradigm performed in duplicate. $^{7}$ The HR assessment paradigm was a 2-condition block de- sign paradigm ( 30 seconds, 12 volumes/condition, 4.5 cycle). The active condition was hand clasping paced $(0.3 \mathrm{~Hz})$ by simultaneous binaural commands (open, close) and central visual commands (OPEN, CLOSE) with peripheral flashing checkerboards (black and white, alternating checkerboard, $10 \mathrm{~Hz}$ ). The fixation condition was a static central white cross on a black background. Patients were trained with this paradigm immediately before the examination. Head alignment and eye movement were monitored during imaging.

BOLD activation was detected with a voxelwise 2-tailed $t$ test as the difference in image signal intensities between the 2 conditions averaged across the 4 cycles at multiple $t$ thresholds with and without a small Gaussian filter (full width at half maximum $=3$ $\mathrm{mm})$. The threshold value ( $t$ value $=3.0$ ) was empirically selected to show $<5 \%$ active voxels outside the gray matter. BOLD activation was superimposed over coregistered high-resolution T1weighted anatomic images for localization of activation in each vascular territory.

The expected activation areas were the supplementary motor areas supplied by the anterior cerebral arteries (ACAs) along the medial frontal lobes; primary sensorimotor areas supplied by the superior middle cerebral artery (sMCA) along the pre- and postcentral gyri; the primary auditory cortex supplied by the inferior MCA along the superior temporal gyri; and the primary and associative visual cortices supplied by the posterior cerebral artery (PCA) along the calcarine fissures of the occipital lobes. Each territory was labeled in a binary fashion as positive or negative for BOLD contrast without regard for asymmetry in activation at the common threshold, indicating intact (+BOLD) or deficient (-BOLD) HR, respectively.

\section{DSC Perfusion MR Imaging}

The DSC perfusion imaging was performed at $3 \mathrm{~T}$ using the previously published DSC perfusion protocol ${ }^{7}$ with a standard body weight-based dose $(0.1 \mathrm{mmol} / \mathrm{kg})$ of intravenous gadoliniumbased contrast (Omniscan; GE Healthcare, Piscataway, New Jersey) followed by a saline flush $(20 \mathrm{~mL})$ delivered by a mechanical injector $(5 \mathrm{~mL} / \mathrm{s}$, delay 10 seconds, 20-ga access; Medrad, Indianola, Pennsylvania) through a large vein in the anticubital fossa of either arm. ${ }^{7}$ After an initial perfusion study (pre-acetazolamide), vasodilation was induced using intravenous administration of acetazolamide (Diamox, $1 \mathrm{~g}$ administered intravenously over 5 minutes). The repeat DSC perfusion imaging was performed after a further delay of 10 minutes to allow full vasodilation.

Time course data were analyzed by a voxelwise fitting of a $\gamma$ variate function to the contrast agent concentration-time-series using a multivariate nonlinear least-squares fitting algorithm as described in detail elsewhere. ${ }^{8,9}$ The fitted points were limited to the initial baseline, leading edge, and the initial portion of the trailing edge after the peak ( $<4$ points) to avoid contamination from contrast recirculation. Although other methods may be used, ${ }^{10}$ this $\gamma$ variate method avoids bias from the choice of the arterial input function in the setting of CVD. ${ }^{11,12}$ The MTT was defined as the first moment of the $\gamma$ variate function fitted to the contrast agent concentration-time-series. The relative CBV was the integral of the $\gamma$ variate function. Only the results for MTT are 
shown because the CBV replicated the same responses. The perfusion parameters (MTT, CBV) were obtained pre- and postvasodilation from ROIs placed in the expected gray matter of fMRI activation in the vascular territory of each cerebral artery using the aligned high-resolution anatomic image (Fig 1). Each ROI contained at least 15 voxels and had a minimum contrast-to-noise ratio of 5. The means and SDs of each perfusion parameter were compared across vascular territories both before and after vasodilation. For SVDd and SVDp, the mean perfusion parameters were compared between cerebral hemispheres (4 left-right comparisons). For patients with $\mathrm{MMD}$, the anterior vascular territories (ACA, sMCA, inferior division of the MCA) were compared with the ipsilateral PCA territories (6 anteroposterior comparisons). Depending on the complexity and distribution of disease in patients with MVD, either left-right or anterior-posterior comparisons were used between vascular territories with and without proximal disease.

Two-tailed Student $t$ tests were used to detect statistically significant differences $(P<.05)$ in perfusion parameters in each pair of vascular territories in each patient, both before and after acetazolamide. The differences in perfusion parameters from pre-acetazolamide to post-acetazolamide in the paired vascular territories were then examined for statistical significance using a second 2-tailed Student $t$ test $(P<.05)$.

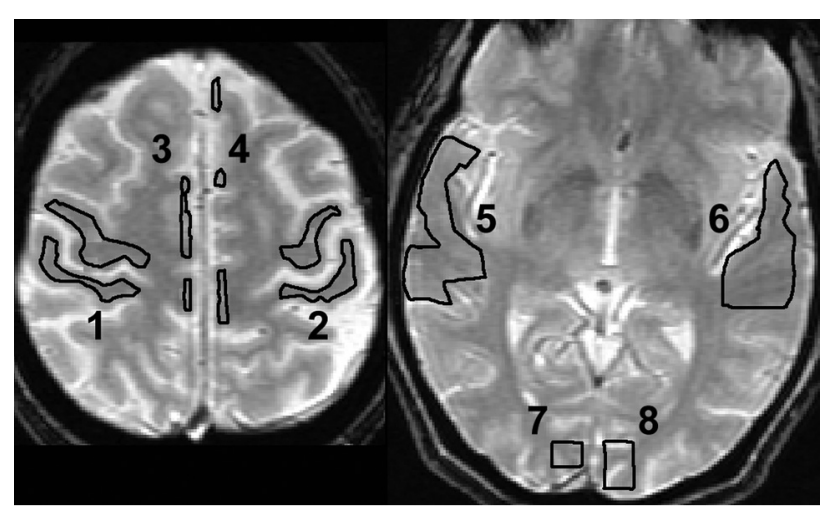

FIG 1. ROls were drawn manually on anatomic T2-weighted images coregistered with the perfusion images in each of the vascular territories in which regional BOLD activation is expected anatomically (1 and 2: right and left primary sensorimotor areas; 3 and 4: right and left supplementary motor areas; 5 and 6 : right and left primary auditory areas; and 7 and 8: right and left primary visual areas). Voxels were included in the ROI if they were $>50 \%$ within the boundary.

\section{Digital Subtraction Angiography}

DSA was used to define the group (SVDd, SVDp, MVD, MMD) for each patient. Vascular narrowing in any vessel was sufficient for consideration to enter this study. These DSA studies were used in all patients to classify the extent of collateral circulation in the region distal to the compromised vessel on a $0-3$ scale by an experienced neurointerventionalist using a previously described grading scale, ${ }^{13}$ in which 3 represents extensive leptomeningeal collateral circulation.

\section{Concordance Analysis}

The perfusion analysis was performed between paired vascular territories with normal and stenotic supply vessels. Territories labeled $+\Delta$ MTT showed an increasing difference in MTT between paired territories after vasodilation, conventionally implying loss of HR. In contrast, $-\Delta$ MTT meant intact HR without statistically significant increases in the difference in MTT following vasodilation. BOLD fMRI used - BOLD to indicate loss of BOLD contrast, implying insufficient HR to support BOLD contrast at the global threshold in a given vascular territory. Intact HR sufficient to support BOLD contrast was indicated by + BOLD. The resultant contingency tables were examined for concordance between the 2 methods by the Fisher exact probability test (Table). This test provided a $P$ value $(P<$ $.05)$ to indicate the risk of being wrong for rejecting the null hypothesis that the 2 methods were equivalent. Concordance was also examined for 2 subgroups of the MMD group showing different scores for leptomeningeal collateral circulation (LCC score $\leq 2$ and LCC score 3 ). Other groups had scores of 3 in most vascular territories. The alternative parameter, proportions of agreement (poa), was used to indicate fractional agreement between methods.

\section{Modeling}

The $\gamma$ variate function is the functional form of the shape of the contrast agent concentration-time curve at the capillary bed. A voxel in tissue with no proximal vascular disease has a single AIF, and there is no pressure differential to establish collateral circulation among the different vascular territories (Fig 2, left). If critical stenosis develops proximally in 1 vessel, its distal capillary bed dilates to generate a pressure differential to adjacent vascular territories to open flow through existing leptomeningeal collateral vessels (Fig 2, right). The AIFs from these adjacent vessels are displaced in time by the increased lengths of the collateral pathways. The total perfusion of the voxel distal to the stenosis is now

\begin{tabular}{|c|c|c|c|c|c|}
\hline CVD Group & $\begin{array}{l}\text { Number of } \\
\text { Cases }\end{array}$ & $\begin{array}{l}\text { Territories } \\
\text { Distal to } \\
\text { Disease }\end{array}$ & $\begin{array}{l}\text { Number of } \\
\text { Territories }\end{array}$ & $\begin{array}{l}\text { Null Hypothesis, } \\
\text { Fisher Exact }\end{array}$ & $\begin{array}{c}\text { Proportions of } \\
\text { Agreement } \\
( \pm 95 \% \mathrm{Cl})\end{array}$ \\
\hline \multirow[t]{2}{*}{ SVDd } & 7 & No & 14 & Accept $(P=1)$ & $0.93(0.642-0.99)$ \\
\hline & & Yes & 10 & Accept $(P=1)$ & $0.70(0.35-0.89)$ \\
\hline \multirow[t]{2}{*}{ SVDp } & 9 & No & 9 & Accept $(P=1)$ & $0.89(0.51-0.99)$ \\
\hline & & Yes & 23 & Accept $(P=.96)$ & $0.91(0.71-0.99)$ \\
\hline \multirow[t]{2}{*}{ MVD } & 10 & No & 20 & Accept $(P=1)$ & $0.90(0.67-0.98)$ \\
\hline & & Yes & 35 & Reject $(P<.004)$ & $0.77(0.51-0.86)$ \\
\hline MMD (LCC $\leq 2)$ & 8 & Yes & 46 & Accept $(P=.41)$ & $0.46(0.31-0.61)$ \\
\hline MMD (LCC = 3) & 6 & Yes & 36 & Reject $(P<.004)$ & $0.72(0.24-0.57)$ \\
\hline
\end{tabular}

${ }^{a}$ Vascular territories with stroke were excluded from comparisons. 


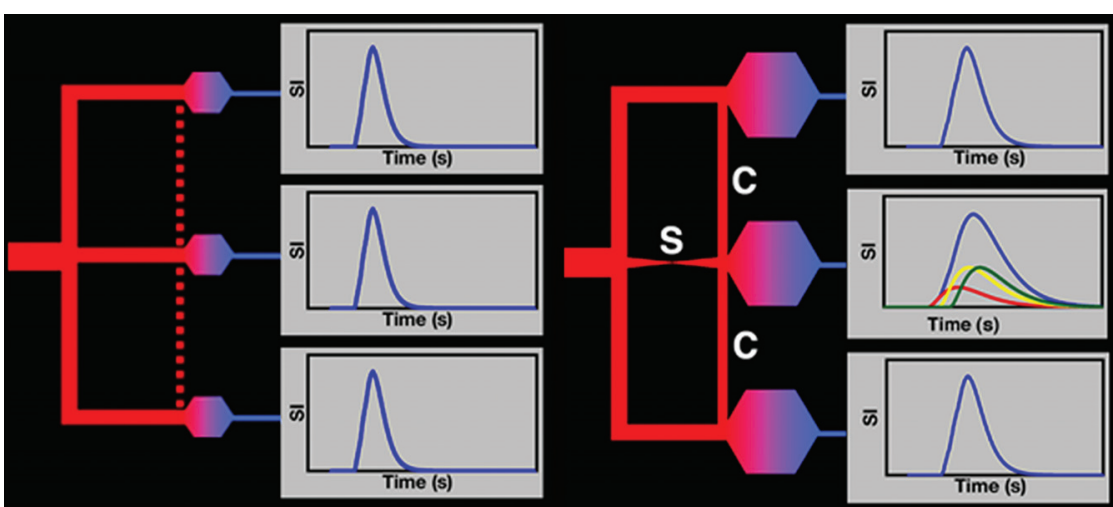

FIG 2. Left: Contrast agent concentration-time curves (blue curves) for 3 vascular territories (upper, middle, lower) are similar for normal vessels. No pressure differential exists between vascular territories to open potential leptomeningeal collateral vessels (dotted red line). Right: Stenosis (S) of the middle territory causes distal dilation, producing a pressure differential that opens leptomeningeal collateral vessels (C) from neighboring vascular territories (upper, lower). The contrast agent concentration-time curve of the compromised territory (middle blue curve) becomes the sum of 3 unresolved inputs (red, yellow, and green curves) temporally displaced due to the increased vascular path lengths from the other territories. MTT lengthens in the compromised territory (temporally displaced middle blue curve compared with the upper and lower blue curves). Pharmacologic vasodilation dilates all capillary beds, thereby decreasing the pressure difference between normal and compromised territories to further delay collateral leptomeningeal flow and increase the temporal separations of the multiple AIFs to lengthen MTT but not necessarily reflecting inadequate flow.

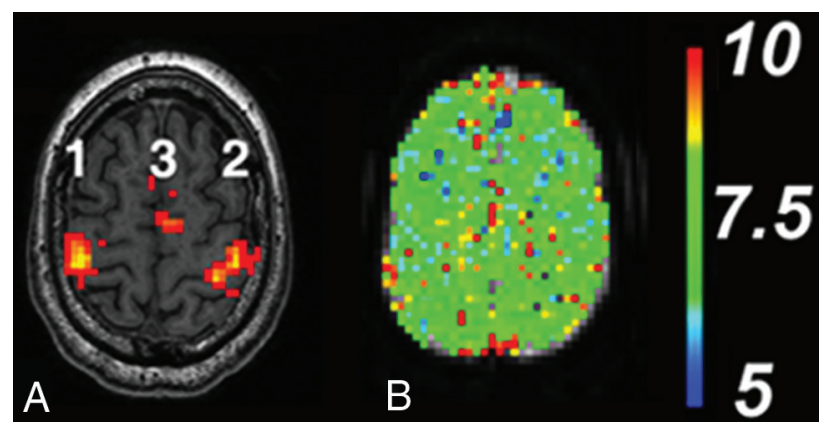

FIG 3. $A$, Normal sensorimotor activation pattern for BOLD fMRI with the HR assessment paradigm. Areas of activation are the following: 1) right primary sensorimotor cortex (right sMCA), 2) left primary sensorimotor cortex (left sMCA), and 3) left and right supplementary motor area (left and right ACA, respectively). The color scale reflects the continuous $t$ value (threshold $t=3$ red, $t>3$ yellow) of the activation-detection statistics. $B$, Normal quantitative perfusion map of MTT at the same level as $A$ remains symmetric before and after vasodilation. The color scale is in seconds.

the sum of 3 AIFs. This combined AIF also has the form of a $\gamma$ variate function but with a longer MTT due to the temporal displacements of the collateral AIF. When the capillary beds are dilated pharmacologically, more blood flow enters the 2 normal vessels because their capillary beds now have decreased resistance. If the cardiac output is sufficient, the collateral leptomeningeal circulation remains intact but with reduced flow due to the reduced pressure differential between the normal and compromised vascular territories. The result is an even longer MTT in the territory distal to the stenotic vessel. The temporal spacing and change in magnitudes of the delayed collateral AIF further lengthen the MTT. This pattern may be better termed "redistribution" than "steal" because the tissue in the compromised territory may still receive adequate total flow to maintain metabolism.

\section{RESULTS}

Representative patterns of BOLD activation for the HR assessment paradigm and for normal perfusion MTT preand postvasodilation in the primary sensorimotor cortex for subjects without CVD are shown in Fig 3. The BOLD pattern shows activation in the supplementary motor and primary sensorimotor areas. The primary auditory and primary visual and visual association areas (not shown) are equally robust. The perfusion maps without vascular disease are reproducible preand postvasodilation and symmetric in both left-right and anteroposterior comparisons.

All CVD cases showed prominent collateral circulation (grade 2 or 3 ) documented by DSA $(n=40)$ and tabulated as LCC scores in On-line Tables 1 and 2. The MTT values for all vascular territories for every subject in all groups are provided in On-line Tables 3 and 4. Representative examples of concordant and nonconcordant patterns for BOLD fMRI and provocative DSC perfusion are shown in Figs 4 and 5, respectively. The statistical parameters and confidence intervals for concordance between the 2 methods across the 4 CVD categories in vascular territories with normal and diseased supply vessels are shown in the Table. For the SVDd and SVDp, the Fisher exact probability test indicated that the null hypothesis of the 2 methods being in agreement must be accepted in both normal and poststenotic vascular territories. However, this null hypothesis should be rejected for the MVD and MMD (LCC score $=3$ ) with extensive leptomeningeal collateral circulation. For vascular territories without stenosis in the MVD group and for MMD (LCC score $\leq 2$ ) with less extensive leptomeningeal collateral circulation, the null hypothesis also cannot be rejected.

However, the proportions of agreement parameter offers additional insight into these methods for assessing HR, indicating that they are more often in agreement in vascular territories without vascular disease distal to the circle of Willis (SVDd, poa = 0.93; SVDp, poa $=0.89$, MVD, poa $=0.90$ ) than when vascular stenosis distal to the circle of Willis is present (SVDd, poa $=0.70$; MVD, poa $=0.77)$. The presence of vascular disease produces a greater number of territories with compromised HR by DSC perfusion MR imaging than by fMRI. When the vascular disease is limited to a unilateral internal carotid artery proximal to the circle of Willis (SVDp), no difference in the 2 methods is detected (territories contralateral to stenotic ICA, poa $=0.89$; territories ipsilateral to stenotic ICA, poa $=0.91)$. Although the null hypothesis cannot be rejected for the MMD group with less extensive leptomeningeal disease (LCC scores $\leq 2$ ), agreement between the 2 methods was low (poa $=0.46$ ), again with perfusion showing increasing MTT differences in the presence of BOLD activation.

AJNR Am J Neuroradiol 39:448-53 Mar 2018 www.ajnr.org 


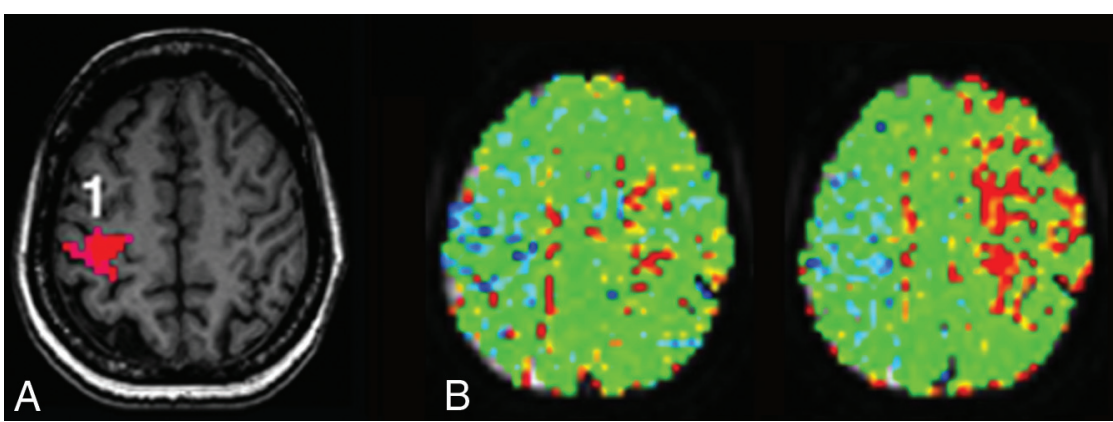

FIG 4. Representative patient with MMD showing concordance. $A, B O L D$ fMRI shows activation in the right (1) but not the left primary sensorimotor area or supplementary motor areas bilaterally. The task was performed correctly because bilateral cerebellar motor and visual activations were present (not shown). B, Quantitative MTT maps through the same level as in $A$ before (left) and after (right) vasodilation. The lengthening of the MTT values after vasodilation would normally be interpreted as loss of HR. The color scale is same as in Fig 3.

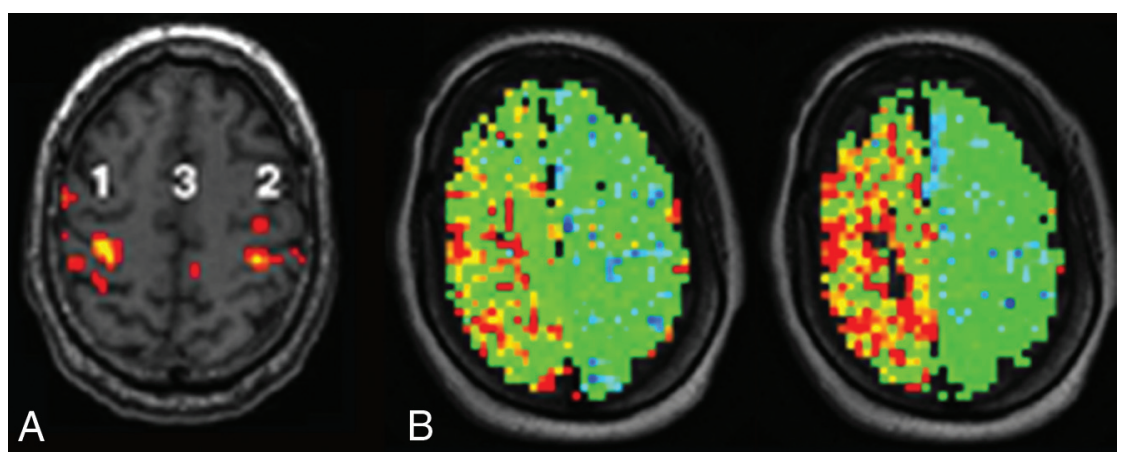

FIG 5. Representative patient with single-vessel disease with unilateral intracranial right internal carotid artery stenosis showing lack of concordance. A, BOLD fMRI activation is intact bilaterally in the primary sensorimotor cortex (1 and 2) and supplementary motor cortex (3) as were auditory and visual cortex activations (not shown). B, MTT maps through the sensorimotor cortex show increasing MTT asymmetry from before (left) to after vasodilation (right), conventionally interpreted as loss of HR. The color scale is same as in Fig 3.

\section{DISCUSSION}

The management of chronic CVD to avoid future stroke involves selecting from multiple interventions that progress from lifestyle changes to more aggressive medical management and ultimately to neurosurgical approaches of angioplasty, stents, and vascular reconstruction. The choice depends on the risk of future stroke because each treatment has financial costs, compliance issues, and potential complications.

Our clinical experience of low concordance between the 2 methods in patients with chronic CVD reflects the results of this retrospective study using technically adequate imaging studies (Table). Extensive collateral leptomeningeal circulation was present anatomically in all patients, but the adequacy of such collateral circulation in the distal tissue cannot be readily assessed angiographically. Perfusion, in which greater asymmetries in MTT following vasodilation would conventionally be interpreted as "steal" or loss of HR, frequently did not agree with intact BOLD fMRI activation in the same vascular territories. Because the perfusion method alone is being advocated as a means for making surgical decisions for intervention in Moyamoya disease, ${ }^{14}$ this discrepancy must be understood.

The results summarized in the Table indicate that both methods are concordant in the absence of disease and even in the setting of disease in which the circle of Willis can provide collateral flow through short large diameter vessels into the proximal cerebral arteries (SVDp). Concordance decreases when the cerebral arteries are compromised distal to the circle of Willis (SCDd) when leptomeningeal vessels must compensate for reduced flow through the stenotic vessel, albeit with temporally displaced arterial inputs, to supply the vascular territory (MMD, MVD). Increasing leptomeningeal circulation in MMD implies decreasing flow from central vessels. The increased concordance for the higher LCC-scored MMD cohort may be attributed to less temporal displacement of AIF when most flow is leptomeningeal rather than from central vessels.

The model for analysis of nondiffusible contrast agents in DSC perfusion assumes that all voxels have a common global arterial input function. ${ }^{10-12}$ In CVD, the substantial collateral pathways are longer and smaller, so the AIF can be displaced temporally from that of the stenotic cerebral arteries. The multiple temporally displaced arterial inputs from both primary and collateral leptomeningeal vessels may become more exaggerated with vasodilation. Fig 2 illustrates how multiple temporally displaced arterial inputs produce prolonged MTT, possibly unrelated to the adequacy of supply. Such increasing asymmetry compared with the normal flow pattern represents redistribution - that is, a change in the perfusion pattern not necessarily reflecting the adequacy of oxygen delivery to the tissue and still having sufficient HR to support BOLD activation.

Conversely, the perfusion parameters may be sufficiently perturbed at baseline to decrease sensitivity to further changes following vasodilation. The complexity of collateral circulation in severe intracranial disease may make the perfusion parameters insensitive to the metabolic demands of the tissue. This substantial collateral circulation would rationalize the lower concordance with the BOLD technique in chronic intracranial CVD.

The limitations of this study are the small number of subjects in each group because of the strict selection criteria. Patients with CVD do not appear to tolerate extended MR imaging studies without head motion. Although the fMRI paradigm robustly produces BOLD contrast in well-established cortical locations of each cerebral artery, it remains sensitive to head motion easily recognized as falsely positive BOLD activation along high-contrast CSF-tissue margins of the image rather than at expected locations. This problem was eliminated from this study cohort by the selection criteria. Vascular territories with areas of encephalomalacic changes from previous strokes or hemorrhage do not show activation, but such vascular territories were excluded from this anal- 
ysis by the selection criteria. The statistical threshold for activation was arbitrarily selected to minimize such false-positive activation in white matter. Activation was considered present only when colocalized to the specific cortical pattern established in healthy subjects. Any asymmetry in activation was ignored. The BOLD technique has the additional disadvantage of being a regional measure that may miss smaller regions of compromised perfusion away from the areas of activation. This limitation did not affect these results because concordance was measured in the same areas of eloquent cortex. The role of global neurovascular reactivity with a hypercapnia challenge may be a means to generalize the task-based fMRI regional response into a global response. $^{15}$

\section{CONCLUSIONS}

The low concordance between BOLD fMRI and provocative perfusion MR imaging for detecting HR compromise in potentially compromised vascular territories of patients with chronic CVD can be rationalized by the complex arterial supply provided by the leptomeningeal collateral circulation that results in temporally displaced multiple arterial inputs. Care is advised when using the perfusion methodology alone to manage patients with complex CVD.

Disclosures: Keith R. Thulborn-RELATED: Grant: National Institutes of Health, Comments: RO1 NS059745-01A1, RO1 NS386760-1A1, and RO1 CA129553-1A1*. Ian C. Atkinson-UNRELATED: Consultancy: Thulborn Associates. Ali Alaraj-UNRELATED: Consultancy: Cordis-Codman. Fady T. Charbel—UNRELATED: Board Membership: Vassol, Comments: noncompensated position; Grants/Grants Pending: National Institute of Neurological Disorders and Stroke*; Patents (Planned, Pending or Issued): Vassol*; Royalties: Vassol*; Stock/Stock Options: Vassol. *Money paid to the institution.

\section{REFERENCES}

1. Gupta A, Chazen JL, Hartman M, et al. Cerebrovascular reserve and stroke risk in patients with carotid stenosis or occlusion: a systematic review and meta-analysis. Stroke 2012;43:2884-91 CrossRef Medline

2. Vagal AS, Leach JL, Fernandez-Ulloa M, et al. The acetazolamide challenge: techniques and applications in the evaluation of chronic cerebral ischemia. AJNR Am J Neuroradiol 2009;30:876-84 CrossRef Medline
3. Mandell DM, Han JS, Poublanc J, et al. Mapping cerebrovascular reactivity using blood oxygen level-dependent MRI in patients with arterial steno-occlusive disease: comparison with arterial spin labeling MRI. Stroke 2008;39:2021-28 CrossRef Medline

4. Derdeyn CP, Videen TO, Yundt KD, et al. Variability of cerebral blood volume and oxygen extraction: stages of cerebral haemodynamic impairment revisited. Brain 2002;125:595-607 CrossRef Medline

5. Huettel SA, Song AW, McCathy G. Functional Magnetic Resonance Imaging. Sunderland: Sinauer Associates, Inc; 2004:128-214

6. Christen T, Bolar DS, Zaharchuk G. Imaging brain oxygenation with MRI using blood oxygenation approaches: methods, validation, and clinical applications. AJNR Am J Neuroradiol 2013;34:1113-23 CrossRef Medline

7. Thulborn KR. Magnetic resonance imaging in the management of cerebrovascular disease to prevent stroke. Neurol Clin 2008;26:897921, vii-viii CrossRef Medline

8. Ostergaard L, Weisskoff RM, Chesler DA, et al. High resolution measurement of cerebral blood flow using intravascular tracer bolus passages, Part I: mathematical approach and statistical analysis. Magn Reson Med 1996;36:715-25 CrossRef Medline

9. Ostergaard L, Sorensen AG, Kwong KK, et al. High resolution measurement of cerebral blood flow using intravascular tracer bolus passages, part II: experimental comparison and preliminary results. Magn Reson Med 1996;36:726-36 CrossRef Medline

10. Calamante F. Bolus dispersion issues related to the quantification of perfusion MRI data. J Magn Reson Imaging 2005;22: 718-22 CrossRef Medline

11. Axel L. Cerebral blood flow determination by rapid-sequence computed tomography: theoretical analysis. Radiology 1980;137:679-86 CrossRef Medline

12. Patil V, Johnson G. An improved model for describing the contrast bolus in perfusion MRI. Med Phys 2011;38:6380-83 CrossRef Medline

13. Kim JJ, Fischbein NJ, Lu Y, et al. Regional angiographic grading system for collateral flow: correlation with cerebral infarction in patients with middle cerebral artery occlusion. Stroke 2004;35: 1340-44 CrossRef Medline

14. Ishii Y, Nariai T, Tanaka Y, et al. Practical clinical use of dynamic susceptibility contrast magnetic resonance imaging (DSC-MRI) for the surgical treatment of moyamoya disease. Neurosurgery 2014;74: 302-09 CrossRef Medline

15. Prisman E, Slessarev M, Han J, et al. Comparison of the effects of independently-controlled end-tidal $\operatorname{PCO}(2)$ and $\operatorname{PO}(2)$ on blood oxygen level-dependent (BOLD) MRI. J Magn Reson Imaging 2008; 27:185-91 CrossRef Medline 